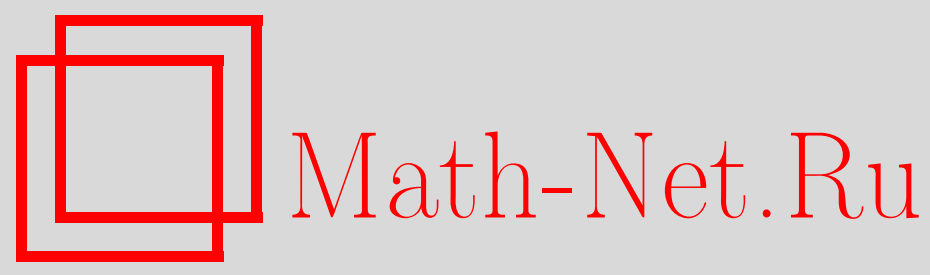

Н. Н. Метельский, В. Г. Найденко, Алгоритмические аспекты частичной выпуклости, Матем. заметки, 2000, том 68, выпуск 3, 399-410

DOI: https://doi.org/10.4213/mzm957

Использование Общероссийского математического портала Math-Net.Ru подразумевает, что вы прочитали и согласны с пользовательским соглашением http://www . mathnet.ru/rus/agreement

Параметры загрузки:

IP : 52.87 .193 .239

26 апреля 2023 г., 08:31:17

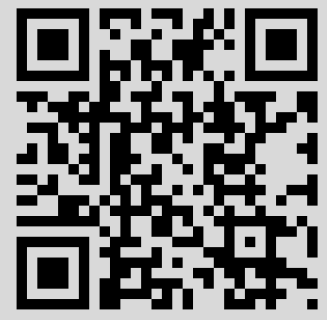




\title{
АЛГОРИТМИЧЕСКИЕ АСПЕКТЫ ЧАСТИЧНОЙ ВЫПУКЛОСТИ
}

\author{
Н. Н. Метельский, В.Г. Найденко
}

Получена характеризация $C$-семипространств частичной вьпуклости. Приведена оценка числа $C$-семипространств в случае, когда число направлений частичной выпуклости конечно. Разработан алгоритм перечисления $C$-семипространств с полиномиальной скоростью. Доказано, что проблема распознавания аппроксимаций частично-выпуклых оболочек, порожденных пересечением $C$-семипространств частичной выпуклости, NP-трудна.

Библиограффия: 5 названий.

В работе [1] был выделен один специальньй класс семипространств частичной выпуклости, так называемые $C$-семипространства. В настоящей статье исследуются свойства $C$-семипространств и проблема нахождения частично-вьпуклых оболочек, порождаемых $C$-семипространствами. Кроме того, мы рассматриваем один вопрос И. Матоушека и П. Плехача о вычислительной сложности построения обобщенных выпуклых оболочек [2].

Пусть в $n$-мерном линейном пространстве $\mathbb{R}^{n}$ задано множество единичных векторов $O \subseteq S^{n-1}$ (направлений), где $S^{n-1}$ - единичная сфера. Мы предполагаем симметричность множества $O$, т.е. $O=-O$, что не является ограничением в контексте частичной вьпуклости. Напомним, что множество $X \subseteq \mathbb{R}^{n}$ назьвается частично-выпуклым $(O$-выпуклым $)$, если пересечение $X$ с произвольной прямой с направляющим вектором из $O$ связно или пусто. Семипространством $S_{a}$ в точке $a \in \mathbb{R}^{n}$ назьвается максимальное по включению вьпуклое (вообще говоря, обобщенно вьпуклое) множество, не содержащее $a$. Чтобы не возникло путанищы, семипространство классической вьпуклости будем обозначать символом $S_{a}$, а семипространство частичной выпуклости чеpeз $S_{a}^{o}$.

Множество $M \subset O$ назовем половинным, если $M \cup-M=O$ и $M \cap-M=\varnothing$. Под выпуклым конусом мы понимаем множество $C$, содержащее $\lambda x+\mu y$ для любых

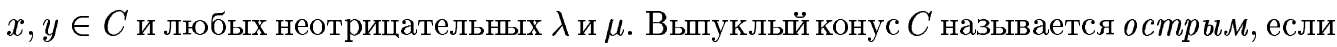
$C$ не содержит одномерных подпространств, т.е. $x \in C$ и $-x \in C$ влечет $x=\mathbf{0}$.

Через $C H[X]$ обозначим вьпуклую коническую оболочку множества $X \subseteq \mathbb{R}^{n}$, т.е. наименьший выпукльй конус, содержаший $X$. Также будем говорить, что конус $C$ noрождается множеством $X$, если $C=C H[X]$.

Работа профинансирована Институтом математики НАН Беларуси в рамках Государственной программы фундаментальных исследований "Алгоритм" при частичной поддержке Фонда фундаментальных исследований Республики Беларусь, гранты Ф97-205 и Ф98М-033. 
В работе [1] доказано, что если $C$ - некоторьй максимальньй среди острых конусов, порождаемых подмножествами множества $O$, то множество $S_{a}^{o}=\mathbb{R}^{n} \backslash(a+C)$ является семипространством $O$-вьпуклости в точке $a \in \mathbb{R}^{n}$. Семипространства вида $\mathbb{R}^{n} \backslash(a+C)$ назьваются $C$-семипространствами.

Справедливы следующие лемма 1 и теоремы 1 и 2 , характеризующие $C$-семипространства.

ЛЕмма 1. Если выпуклый конус $C$ содержит некоторое половинное множество векторов $M \subset O$, то С включает в себя хотя бы один максимальный острый конус $C H\left[M^{\prime}\right]$, порожденный некоторым подмножеством $M^{\prime} \subset O$.

ДокАЗАТЕЛЬСТво. Ясно, что $C H[M] \subseteq C$. Если $C H[M]-$ острый конус, то $C H[M]$ будет максимальным. Следовательно, $M^{\prime}=M$. Рассмотрим случай, когда $C H[M]-$ не острый. Пусть $X \subset M$ - множество векторов таких, что если $v \in X$, то $v \in C H[M]$ и $-v \notin C H[M]$. Если $X=\varnothing$, то для любого $v \in M$ справедливо $v \in C H[M]$ и $-v \in C H[M]$. А поскольку $M \cup-M=O$, то $C H[M]=C H[O]$. Тогда в качестве $M^{\prime}$ выберем любое подмножество, образующее максимальный острьй конус (в силу леммы 1 из [1] это всегда можно сделать). Рассмотрим случай, когда $X \neq \varnothing$. Отметим, что $C H[X]$ - острьй конус. Следуя работе [1], обозначим через $P C O N(O \mid X)$ множество всех частично упорядоченных по включению острых конусов вида $C H\left[A_{X}\right]$, ге $A_{X} \subseteq O$ такое подмножество, что $X \subseteq C H\left[A_{X}\right]$. Покажем, что множество $P C O N(O \mid X)$ содержит максимальные элементы, которые будут максимальными и для $P C O N(O)$ $(P C O N(O)$ - множество всех острых конусов, порожденных подмножествами из $O$ и частично упорядоченных по включению). Действительно, пусть $\left\{C H\left[A_{X}^{\alpha}\right]\right\}_{\alpha}-$ произвольная цепь элементов, упорядоченных по включению, из $\operatorname{PCON}(O \mid X)$. Тогда $\bigcup_{\alpha}\left\{C H\left[A_{X}^{\alpha}\right]\right\}_{\alpha}$ будет точной верхней гранью, принадлежащей $P C O N(O \mid X)$. Согласно лемме Цорна получаем, что $\operatorname{PCON}(O \mid X)$ имеет максимальные элементы. Заметим, что $P C O N(O \mid X) \subseteq P C O N(O)$. Пусть $C H\left[A_{X}^{*}\right]$ - некоторый максимальный элемент для $P C O N(O \mid X)$, но немаксимальный для $P C O N(O)$. Тогда найдется конус $C H[A] \in P C O N(O)$ такой, что $C H\left[A_{X}^{*}\right] \subset C H[A]$. Но $X \subseteq C H\left[A_{X}^{*}\right]$; следовательно, $X \subset C H[A]$ и $C H[A] \in P C O N(O \mid X)$. Получаем противоречие. Итак, максимальньй элемент для $P C O N(O \mid X)$ будет максимальным и для $P C O N(O)$. Таким образом, существует подмножество $M^{\prime} \subseteq O$ такое, что $C H\left[M^{\prime}\right] \in P C O N(O \mid X)$ и $C H\left[M^{\prime}\right]-$ максимальньй острый конус. Докажем, что $M^{\prime}$ удовлетворяет данной лемме. Поскольку $M \cup-M=O,-X \cap C H[M]=\varnothing$ и $X \subset C H[M]$, то $O \backslash-X \subseteq C H[M]$. Кроме того, $X \subseteq C H\left[M^{\prime}\right]$. Тогда $-X \cap C H\left[M^{\prime}\right]=\varnothing$. Поэтому $M^{\prime} \cap-X=\varnothing$, ибо в противном случае конус $C H\left[M^{\prime}\right]$ не острьй. Но тогда $M^{\prime} \subseteq O \backslash-X$ и, следовательно, $C H\left[M^{\prime}\right] \subseteq C H[M] \subseteq C$. Лемма доказана.

Через conv $[X]$ будем обозначать вьпуклую (в классическом смысле) оболочку произвольного множества $X \subseteq \mathbb{R}^{n}$.

Tеорема 1. Семипространство О-выпуклости является C-семипространством тогда и только тогда, когда его дополнение относительно $\mathbb{R}^{n}$ выпукло в классическом смысле.

ДокАЗАТЕЛЬСТво. По определению дополнение $C$-семипространства классически выпукло. Осталось доказать, что если дополнение семипространства $O$-вьпуклости 
выпукло, то оно представляет собой $C$-семипространство. Докажем это для семипространств $O$-выпуклости в точке 0 (для семипространств $O$-выпуклости в произвольной точке $x \in \mathbb{R}^{n}$ результат будет таким же, поскольку любое семипространство $S_{x}^{o}$ можно получить параллельньм переносом из точки $\mathbf{0}$ в точку $x$ некоторого семипространства $\left.S_{0}^{o}\right)$. Пересечение любой прямой $l_{v}=\{\lambda v \mid \lambda \in \mathbb{R}\}$, где $v \in O$ - направляющий вектор прямой $l_{v}$, с $\mathbb{R}^{n} \backslash S_{0}^{o}$ должно быть непусто и в силу классической вьпуклости $\mathbb{R}^{n} \backslash S_{0}^{o}$ должно представлять собой точку, отрезок, луч или прямую. Заметим, что $l_{v} \cap\left(\mathbb{R}^{n} \backslash S_{0}^{o}\right)$ не может быть точкой или отрезком, так как в противном случае $l_{v} \cap S_{0}^{o}$ является объединением двух несвязных интервалов, что противоречит $O$-выпуклости $S_{0}^{o}$. Таким образом, $l_{v} \cap\left(\mathbb{R}^{n} \backslash S_{0}^{o}\right)$ является лучом или прямой. Для любого $l_{v} \cap\left(\mathbb{R}^{n} \backslash S_{0}^{o}\right)$ найдется замкнутьй луч $r_{x} \subseteq l_{v} \cap\left(\mathbb{R}^{n} \backslash S_{0}^{o}\right)$, выходящий из точки 0 и совпадающий по направлению с некоторым вектором $x \in\{-v, v\}$. Обозначим объединение всех таких лучей через $r\left(S_{0}^{o}\right)$, а множество векторов из $O$, совпадаюших по направлению с лучами из $r\left(S_{0}^{o}\right)$, через $\operatorname{Or}\left(S_{0}^{o}\right)$. Заметим, что $\operatorname{conv}\left[r\left(S_{0}^{o}\right)\right]=C H\left[\operatorname{Or}\left(S_{0}^{o}\right)\right]$, поскольку выпуклая оболочка объединения лучей, выходящих из одной точки, будет выпукльм конусом (см., например, [3]). Для любого $r_{x} \subseteq r\left(S_{0}^{o}\right)$ верно $r_{x} \subseteq \mathbb{R}^{n} \backslash S_{0}^{o}$; следовательно, $C H\left[O r\left(S_{0}^{o}\right)\right] \subseteq \mathbb{R}^{n} \backslash S_{0}^{o}$. Для любого вектора $v \in O$ справедливо хотя бы одно из утверждений: $v \in \operatorname{Or}\left(S_{0}^{o}\right)$ или $-v \in \operatorname{Or}\left(S_{0}^{o}\right)$, так как $l_{v} \cap\left(\mathbb{R}^{n} \backslash S_{0}^{o}\right) \neq \varnothing$. Это означает, что $\operatorname{Or}\left(S_{0}^{o}\right)$ содержит некоторое половинное подмножество векторов $M \subset O$. Согласно лемме 1 конус $C H\left[O r\left(S_{0}^{o}\right)\right]$ будет содержать некоторый максимальньй острьй конус $C H\left[M^{\prime}\right], M^{\prime} \subset O$. Тогда $C H\left[M^{\prime}\right] \subseteq \mathbb{R}^{n} \backslash S_{0}^{o}$ и, значит, $S_{0}^{o} \subseteq \mathbb{R}^{n} \backslash C H\left[M^{\prime}\right]$. Но $\mathbb{R}^{n} \backslash C H\left[M^{\prime}\right]$ само является $C$-семипространством $O$-вьпуклости. Следовательно, семипространство $S_{0}^{o}$ должно совпадать с $\mathbb{R}^{n} \backslash C H\left[M^{\prime}\right]$. Теорема доказана.

Как следствие из теоремы 1 получаем факт, приведенньй в [1], а именно: все семипространства классической вьпуклости являются $C$-семипространствами для множества направлений $O=S^{n-1}$. Действительно, классическую выпуклость можно рассматривать как частичную при $O=S^{n-1}$. Согласно свойству семипространств классической выпуклости их дополнение выпукло, а значит, по теореме 1 они являются $C$-семипространствами.

Отметим также, что не все конические семипространства частичной выпуклости исчерпываются $C$-семипространствами. Например, пусть задано множество направлений $O=\left\{v_{1}, v_{2}, v_{3}, v_{4}\right\}$ (см. рис. 1 ). Тогда в точке $\mathbf{0}$ семипространство $O$-вьпуклости $S_{0}^{o}=\left(\left\{\alpha_{1} v_{1}+\alpha_{2} v_{2} \mid \alpha_{1}, \alpha_{2}>0\right\} \cup\left\{\alpha_{3} v_{3}+\alpha_{4} v_{4} \mid \alpha_{3}, \alpha_{4} \geqslant 0\right\}\right) \backslash\{\mathbf{0}\}$, показанное на рисунке штриховкой, будет коническим, но не $C$-семипространством.

Докажем следующую теорему, которая дает конструктивный способ построения $C$ семипространств.

Теорема 2. Для любого C-семипространства $S_{a}^{o}=\mathbb{R}^{n} \backslash(a+C H[X])$ существует половинное мнохсество $M \subset O$ такое, что $C H[X]=C H[M]$.

ДоКАЗАТЕЛЬСТВО. Поскольку $C H[X]$ является острым конусом, то $X \cap-X=\varnothing$. Если же $X$ еще и половинное множество, то $M=X$. Рассмотрим случай, когда $X$ не половинное. Покажем, что для любого вектора $v \in O$ справедливо следующее утверждение: хотя бы один из векторов $v$ или $-v$ принадлежит $C H[X]$. В противном случае множество $l=\{\lambda v \mid \lambda \in \mathbb{R}, \lambda \neq 0\}$ не содержится в $C H[X]$, а, следовательно, множество $l^{\prime}=\{a+\lambda v \mid \lambda \in \mathbb{R}, \lambda \neq 0\}$ также не содержится в $a+C H[X]$. Значит, семипространство $S_{a}^{o}=\mathbb{R}^{n} \backslash(a+C H[X])$ включает в себя $l^{\prime}$. Но тогда $S_{a}^{o}$ не $O$-вьпукло, так 


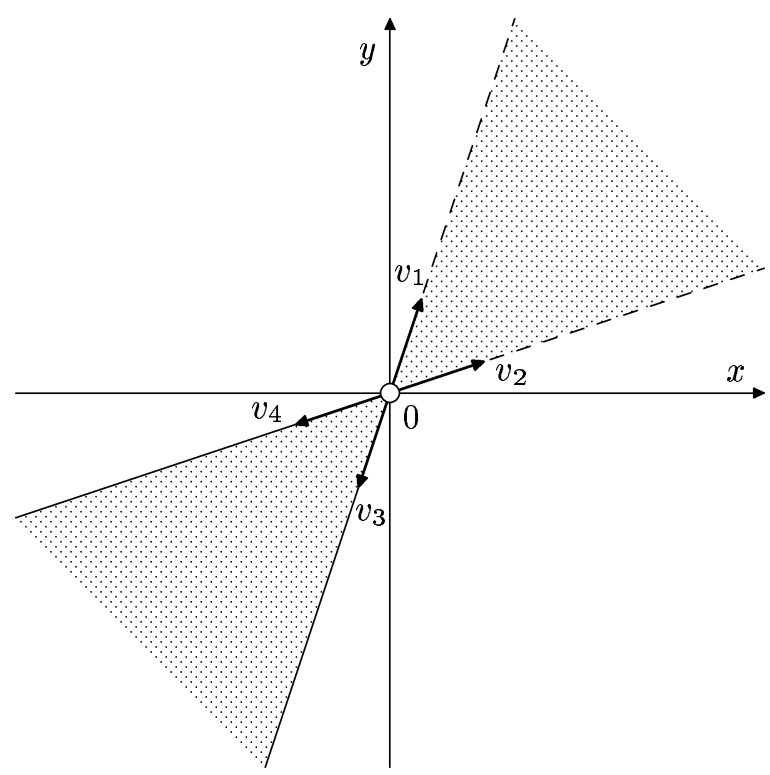

Рис. 1. Пример конического пространства, не являющегося $C$-семипространством

как, например, две точки $a_{1}=a+\lambda v$ и $a_{2}=a-\lambda v$ для некоторого фиксированного $\lambda \neq 0$ принадлежат $S_{a}$, но точка $a=\frac{1}{2} a_{1}+\frac{1}{2} a_{2}$ не содержится в $S_{a}$. Получаем противоречие. Пусть $M$ - множество всех векторов из $O$, принадлежаших $C H[X]$. Тогда в силу выше сказанного $M \cup-M=O$. Заметим также, что $M \cap-M=\varnothing$ в силу остроты конуса $C H[X]$. Значит, $M$ половинное. Так как $M \subseteq C H[X]$, то $C H[M] \subseteq C H[X]$. Но поскольку $X \subseteq M$, то $C H[M]=C H[X]$ и, следовательно, множество $M$ удовлетворяет условию теоремы. Теорема доказана.

Таким образом, можно считать, что все $C$-семипространства, описанные в работе [1], порождаются некоторыми половинными подмножествами множества $O$.

Заметим, что если коническая оболочка $C H[M]$ некоторого половинного множества $M \subset O$ является острым конусом, то конус $C H[M]$ максимальньй по включению среди всех выпуклых острых конических оболочек, порожденных подмножествами множества $O$. Итак, чтобы найти все $C$-семипространства, достаточно перебрать все половинные подмножества множества $O$ и для каждого такого подмножества $M$ проверить, будет ли его коническая оболочка $C H[M]$ острой. Если да, то $S_{a}^{o}=\mathbb{R}^{n} \backslash(a+C H[M])$ будет $C$-семипространством $O$-выпуклости в точке $a$. Если $M$ конечно, то остроту конуса $C H[M]$ можно проверить следуюшим образом. Конус $C H[M]$ будет острым тогда и только тогда, когда система уравнений относительно переменных $\alpha_{1}, \ldots, \alpha_{m} \in \mathbb{R}$

$$
\alpha_{1} v_{1}+\cdots+\alpha_{m} v_{m}=0, \quad \alpha_{1}, \ldots, \alpha_{m} \geqslant 0
$$

где $\left\{v_{1}, \ldots, v_{m}\right\}=M$, будет иметь единственное решение $\alpha_{1}=\alpha_{2}=\cdots=\alpha_{m}=0$. 
Чтобы проверить это, достаточно решить задачу линейного программирования

$$
\begin{gathered}
\sum_{i=1}^{m} \alpha_{i} \rightarrow \max , \\
\alpha_{1} v_{1}+\cdots+\alpha_{m} v_{m}=0, \quad \alpha_{1}, \ldots, \alpha_{m} \geqslant 0 .
\end{gathered}
$$

Если $\sum_{i=1}^{m} \alpha_{i}=0$, то конус $C H[M]$ острый.

Перейдем теперь к алгоритму перечисления всех половинных множеств, конические оболочки которьх - максимальные острые конусы, в случае, когда $O$ конечно.

Пусть $M_{0}=\left\{v_{1}^{0}, \ldots, v_{m}^{0}\right\}$ - некоторое произвольное фиксированное половинное множество векторов из $O\left(C H\left[M_{0}\right]\right.$ - необязательно острьй конус). Тогда все половинные множества векторов из $O$ можно представить в виде кортежей из $m$ элементов вида $\left\langle s_{1}, \ldots, s_{m}\right\rangle$, где $s_{i} \in\left\{-v_{i}^{0}, v_{i}^{0}\right\}$ для любого $i \in \overline{1, m}$. На множестве $O$ зададим частичное упорядочение векторов: $\forall i \in \overline{1, m} v_{i}^{0}>-v_{i}^{0}$. На множестве всех половинных множеств векторов из $O$ введем лексикографический порядок. Пусть заданы два произвольных половинных множества $M_{1}=\left\langle s_{1}^{1}, \ldots, s_{m}^{1}\right\rangle$ и $M_{2}=\left\langle s_{1}^{2}, \ldots, s_{m}^{2}\right\rangle$. Будем считать, что $M_{1}>M_{2}$ тогда и только тогда, когда существует $i$ такое, что $s_{i}^{1}>s_{i}^{2}$, и если $i>1$, то $s_{j}^{1}=s_{j}^{2}$ для любого $j, j<i$, причем $i, j \in \overline{1, m}$.

Теперь формально опишем итерационный алгоритм ENUMCONE перечисления всех половинных множеств, порождающих острые конусы. Переменные алгоритма $s_{1}, \ldots, s_{m}$ определены на множестве векторов $\left\{-v_{1}^{0}, v_{1}^{0}, \ldots,-v_{m}^{0}, v_{m}^{0}\right\}=O$. Переменная $i$ обозначает номер итерации.

Алгоритм ENUMCONE.

ШШаг 1. $i:=1 ; e:=1$.

Шаг 2. Если $C H\left[\left\langle s_{1}, \ldots, s_{e-1}, v_{e}^{0}\right\rangle\right]-$ острьй конус, то $s_{e}:=v_{e}^{0} ;$ иначе $s_{e}:=-v_{e}^{0}$.

Шаг 3. $e:=e+1$.

Шаг 4. Если $e \leqslant m$, то переход к шагу 2 .

Шаг 5. Выдать половинное множество векторов $M_{i}=\left\langle s_{1}, \ldots, s_{m}\right\rangle$.

Шаг 6. $i:=i+1$.

Шаг 7. Найти наибольшее $j, j \in \overline{1, m}$, при котором $s_{j}=v_{j}^{0}$ и $C H\left[\left\langle s_{1}, \ldots, s_{j-1},-s_{j}\right\rangle\right]-$ острый конус. В противном случае остановить ENUMCONE (все требуемые половинные множества найдены).

Шаг 8. $s_{j}:=-v_{j}^{0}$.

Шаг 9. $e:=j+1$.

Шаг 10. Переход к шагу 2.

ЗАмечаниЕ. Для $e=1$ и $j=1$ конусы $C H\left[\left\langle s_{1}, \ldots, s_{e-1}, v_{e}^{0}\right\rangle\right]$ и $C H\left[\left\langle s_{1}, \ldots, s_{j-1}\right.\right.$, $\left.\left.-s_{j}\right\rangle\right]$ обозначают соответственно $C H\left[\left\langle v_{1}^{0}\right\rangle\right]$ и $C H\left[\left\langle-s_{1}\right\rangle\right]$.

Алгоритм ENUMCONE в лексикографическом порядке, начиная с самого большего, перечисляет все половинные множества, порождающие острые конусы. Отметим, что несмотря на то, что общее время работы алгоритма ENUMCONE экспоненциально по $n \cdot|O|$ (напомним, что $n$ - размерность линейного пространства $\mathbb{R}^{n}$ ), однако последовательность $M_{1}, M_{2}, \ldots$ половинных множеств генерируется алгоритмом с полиномиальной скоростью (определение скорости (rate) алгоритма дано в [4]). Более строго это утверждение можно сформулировать в виде теоремы 3. Прежде, чем доказьвать теорему 3 , приведем следующую лемму, гарантирующую корректность шага 2 алгоритма ENUMCONE. 
Лемма 2. Пусть $X=\left\{v_{1}, \ldots, v_{m}\right\}$ - произвольное конечное мнохсество векторов и СН[X] - острый конус. Тогда для любого вектора $v \in S^{n-1}$ справедливо утверждение: хотя бы один из конусов $C H[X \cup\{v\}]$ или $C H[X \cup\{-v\}]$ острый.

ДокАЗАТЕЛЬСтво. Заметим, что так как $C H[X]$ - острьй конус, система уравнений относительно переменных $\alpha_{1}, \ldots, \alpha_{m} \in \mathbb{R}$

$$
\alpha_{1} v_{1}+\cdots+\alpha_{m} v_{m}=0, \quad \alpha_{1}, \ldots, \alpha_{m} \geqslant 0, \quad \alpha_{1}+\cdots+\alpha_{m}>0,
$$

не имеет решений. Предположим, что

$$
v=\alpha_{1} v_{1}+\cdots+\alpha_{m} v_{m}
$$

для некоторых неотрицательных $\alpha_{1}, \ldots, \alpha_{m}$. Тогда, очевидно, что $C H[X \cup\{v\}]=$ $C H[X]$. Аналогично, когда

$$
-v=\alpha_{1} v_{1}+\cdots+\alpha_{m} v_{m},
$$

$C H[X \cup\{-v\}]=C H[X]$. Предположим, что ни $(2)$, ни (3) не выполняются и конусы $C H[X \cup\{v\}]$ и $C H[X \cup\{-v\}]$ не острые. Тогда система уравнений (4) относительно переменных $\alpha, \beta, \alpha_{1}, \ldots, \alpha_{m}, \beta_{1}, \ldots, \beta_{m} \in \mathbb{R}$ имеет решение

$$
\begin{gathered}
\alpha_{1} v_{1}+\cdots+\alpha_{m} v_{m}+\alpha v=0, \\
\beta_{1} v_{1}+\cdots+\beta_{m} v_{m}+\beta(-v)=0, \\
\alpha, \beta, \alpha_{1}, \ldots, \alpha_{m}, \beta_{1}, \ldots, \beta_{m} \geqslant 0, \\
\alpha+\alpha_{1}+\cdots+\alpha_{m}>0, \quad \beta+\beta_{1}+\cdots+\beta_{m}>0 .
\end{gathered}
$$

Из системы (4) следует, что $\alpha_{1} v_{1}+\cdots+\alpha_{m} v_{m}=-\alpha v$ и $\beta_{1} v_{1}+\cdots+\beta_{m} v_{m}=\beta v$. В силу того, что система (1) не имеет решений, $\alpha$ и $\beta$ положительны. То есть конус $C H[X]$ содержит прямую с направляющим вектором $v$, что противоречит принятому ранее условию об остроте $C H[X]$. Таким образом, обязательно один из конусов $C H[X \cup\{v\}]$ или $C H[X \cup\{-v\}]$ будет острьг. Лемма доказана.

TЕОремА 3. Существует полином р такой, что алгоритм ENUMCONE для заданного конечного мнохества направлений $O \subset S^{n-1}$ выводит последовательность $M_{1}, M_{2}, \ldots$ всех половинных множеств векторов из $O$, порождающих максимальные острые конусы, причем каждое множество $M_{i}$ находится за время, не превосходящее $p(n \cdot|O|)$.

ДокАЗАТЕЛЬСтво. Доказательство будем вести индукцией по числу итераций. Любое половинное множество, находимое алгоритмом ENUMCONE, порождает острьй конус в силу леммы 2. Очевидно, что алгоритм ENUMCONE генерирует первое половинное множество $M_{1}$ за полиномиальное время по $n \cdot|O|$. Допустим ENUMCONE на итерации $i$ нашел множество $M_{i}$. Следующее множество $M_{i+1}$ находится за полиномиальное время, так как шаг 7 алгоритма ENUMCONE также можно вьполнить за полиномиальное время. Итак, алгоритм ENUMCONE перечисляет множества $M_{1}, M_{2}, \ldots$ с полиномиальной скоростью. Осталось показать, что алгоритм ENUMCONE перечисляет все без исключения половинные множества, порождающие максимальные острые конусы. Заметим, что $M_{1}$ является наибольшим в лексикографическом порядке множеством, порождающим острый конус. Действительно, если предположить противное, что 
существует $M^{\prime}=\left\langle s_{1}^{\prime}, \ldots, s_{m}^{\prime}\right\rangle$, большее, чем $M_{1}=\left\langle s_{1}^{1}, \ldots, s_{m}^{1}\right\rangle$, то найдется $e$, для которого $s_{e}^{\prime}>s_{e}^{1}$, т.е. $s_{e}^{\prime}=v_{e}^{0}$ и $s_{e}^{1}=-v_{e}^{0}$. Но в таком случае конус $C H\left[\left\langle s_{1}^{1}, \ldots, s_{e-1}^{1}, v_{e}^{0}\right\rangle\right]$ острый и в силу шага 2 алгоритма ENUMCONE $s_{e}^{1}$ должно быть равно $v_{e}^{0}$. Получаем противоречие. Далее покажем, что $M_{i+1}=\left\langle s_{1}^{i+1}, \ldots, s_{m}^{i+1}\right\rangle$ ближайшее в лексикографическом порядке ко множеству $M_{i}=\left\langle s_{1}^{i}, \ldots, s_{m}^{i}\right\rangle$. Допустим противное, что существует ближайшее к $M_{i}$ множество $M^{\prime}=\left\langle s_{1}^{\prime}, \ldots, s_{m}^{\prime}\right\rangle$ и $M^{\prime} \neq M_{i+1}$. Следовательно, $M_{i}>M^{\prime}>M_{i+1}$. Тогда найдутся $j$ и $k$ такие, что $s_{j}^{i}>s_{j}^{i+1}$ и $s_{k}^{i}>s_{k}^{\prime}$; кроме того, для любого $l, l<j, s_{l}^{i}=s_{l}^{i+1}$ и для любого $r, r<k, s_{r}^{i}=s_{r}^{\prime}$. Согласно шагу 7 алгоритма ENUMCONE $j$ должно быть равно $k$. Но в этом случае существует $e$ большее, чем $j$, такое, что $s_{e}^{\prime}>s_{e}^{i+1}$. Поэтому конус $C H\left[\left\langle s_{1}^{i+1}, \ldots, s_{e-1}^{i+1}, v_{e}^{0}\right\rangle\right]$ острьй и согласно шагу 2 алгоритма ENUMCONE $s_{e}^{i+1}$ должно быть равно $v_{e}^{0}$. Получаем противоречие. Очевидно также, что алгоритм ENUMCONE останавливается на шаге 7 только в том случае, если $M_{i}$ - наименьшее в лексикографическом порядке половинное множество, порождающее острьй конус. Итак, алгоритм ENUMCONE перечисляет в лексикографическом порядке все половинные множества, вьпуклые оболочки которых представляют собой острые конусы. Теорема доказана.

Перейдем к оценке количества максимальных острых конусов. Пусть $O$ конечно и $m=|O| / 2$. Обозначим оценку количества максимальных острых конусов в пространстве $\mathbb{R}^{n}$ через $q(n, m)$.

ТЕОремА 4. Имеем $q(n, m) \leqslant 2^{n} m^{(n+1)(n-2) / 2} \partial$ Ля $n \geqslant 3$.

ДокАЗАТЕЛЬСтво. Количество всевозможных гиперплоскостей, проведенных через начало координат, на которые опираются $(n-1)$-мерные грани максимальных острых конусов, очевидно, непревышает $C_{m}^{n-1}$ или, болеегрубо, $m^{n-1}$. Любая $(n-1)$-мерная грань произвольного максимального острого конуса, лежащая в некоторой гиперплоскости $H$, является максимальньм острым конусом для множества направлений $O \cap H$. Тогда количество $(n-1)$-мерных граней всех максимальных конусов в $\mathbb{R}^{n}$ не превосходит $m^{n-1} q(n-1, m)$. Кроме того, каждая $(n-1)$-мерная грань является общей в точности для двух максимальных острых конусов. Заметим, что для $\mathbb{R}^{3}$ количество 2 -мерных граней не превьшает $C_{2 m}^{2}$. Тогда $q(3, m) \leqslant 2 C_{2 m}^{2} \leqslant 8 m^{2}$ Получаем следующую рекуррентную формулу для оценки количества максимальных конусов:

$$
q(3, m) \leqslant 8 m^{2}, \quad q(n, m) \leqslant 2 m^{n-1} q(n-1, m) .
$$

Тогда $q(n, m) \leqslant 2 m^{n-1} 2 m^{n-2} \cdots 2 m^{3} 8 m^{2}=2^{n} m^{(n+1)(n-2) / 2}$. Теорема доказана.

Перейдем теперь к проблеме нахождения частично-вьпуклых оболочек, играющих важную роль в таких приложениях, как кристаллография, проектирование СБИС, базы данных и др.

Определение частично-вьпуклой оболочки аналогично ее классическому варианту. Для произвольного $X \subseteq \mathbb{R}^{n}$ частично-выпуклая оболочка (О-оболочка) $\operatorname{conv}^{o}[X]$ есть пересечение всех частично-вьпуклых множеств, содержащих $X$. Возникает следующая проблема: определить, принадлежит ли произвольная точка $a \in \mathbb{R}^{n}$ к $\operatorname{conv}^{o}[X]$, где $X \subset \mathbb{R}^{n}$ - конечно. Отметим, что в отличие от классической выпуклости алгоритм решения указанной проблемы неизвестен для произвольного $n$. Однако ограничиваясь определенным классом $O$-вьпуклых множеств, мы решаем этот вопрос. 
Введем понятие $O C$-выпуклости. Семейство подмножеств $O C$ из $\mathbb{R}^{n}$ образует $O C$-выпуклость, если $O C$ содержит только следующие множества:

1) $\mathbb{R}^{n}$

2) для любого $a \in \mathbb{R}^{n}$ все $C$-семипространства $O$-выпуклости принадлежат $O C$;

3 ) пересечение любой совокупности $C$-семипространств $O$-выпуклости принадлежит $O C$.

Пара $\left(\mathbb{R}^{n}, O C\right)$ с точки зрения аксиоматической теории выпуклости [5] назьвается пространством OC-выпуклости, а элементы семейства $O C-O C$-выпуклыми множествами. $O C$-вьпуклость можно считать частным случаем $O$-выпуклости, так как все $O C$-выпуклые множества являются $O$-вьпуклыми (обратное, очевидно, не верно, см. рис. 1), однако оно позволяет эффективно строить аппроксимации частично-вьпуклых оболочек. Семипространства $O C$-выпуклости в произвольной точке $a \in \mathbb{R}^{n}$ будем для краткости называть $O C$-семипространствами и будем обозначать символом $S_{a}^{o c}$. Справедлива следующая теорема.

Теорема 5. Для любого а $\in \mathbb{R}^{n}$ всякое ОС-семипространство в точке а является $C$-семипространством О-выпуклости в той же точке $а$.

ДокАЗАТЕЛьСтво. Пусть задана некоторая точка $a \in \mathbb{R}^{n}$ и произвольное $O C$-выпуклое множество $F$, где $a \notin F$. Докажем, что найдется $C$-семипространство $O$-вьпуклости $S_{a}^{o}$ в точке $a$ такое, что $F \subseteq S_{a}^{o}$. Действительно, поскольку $F$ является по определению пересечением некоторых $C$-семипространств и $a \notin F$, то найдется $C$-семипространство $S_{b}^{o}$ такое, что $F \subseteq S_{b}^{o}$ и $a \notin S_{b}^{o}$. Если $a=b$, то $F \subseteq S_{a}^{o}$. Рассмотрим случай, когда $a \neq b$. Пусть $S_{b}^{o}=\mathbb{R}^{n} \backslash(b+C)$. Возьмем $C$-семипространство $S_{a}^{o}=\mathbb{R}^{n} \backslash(a+C)$. Из $a \notin S_{b}^{o}$ следует, что $a \in b+C$. Поэтому $a+C \subset b+C$ (в силу выпуклости конуса $C$ ). Следовательно, $S_{b}^{o} \subset S_{a}^{o}$ и в итоге получаем $F \subset S_{a}^{o}$. Таким образом, любое $O C$-вьпуклое множество, не содержащее некоторую фиксированную точку $a$, содержится в некотором $C$-семипространстве в точке $a$. Поскольку все $C$-семипространства являются семипространствами $O C$-выпуклости (исходя из определения $O C$-выпуклости), то получаем доказательство утверждения теоремы.

Для $O C$-выпуклости сохраняются многие свойства, характерные для $O$-выпуклости, сформулированные в теоремах 6-9.

ТЕОРема 6. Все классически выпуклые множества являются ОС-выпуклыми.

ДокАЗАТЕЛЬСТво. Для каждого семипространства $S_{0}$ классической выпуклости в точке $\mathbf{0}$ существует базис $x_{1}, \ldots, x_{n}$ в $\mathbb{R}^{n}$ такой, что

$$
S_{0}=\left\{x \mid x=\sum_{i=1}^{n} a_{i} x_{i}\right\}
$$

где $\sum_{i=1}^{n} a_{i}^{2}>0$ и первое $a_{i}$, отличное от нуля, положительно. Тогда очевидно, что для любого ненулевого вектора $v$ либо $v$, либо $-v$ (но не одновременно) принадлежит $S_{0}$. Обозначим через $O\left(S_{0}\right)$ множество векторов такое, что $O\left(S_{0}\right)=S_{0} \cap O$. Множество $\mathbb{R}^{n} \backslash\left(S_{0} \cup\{\mathbf{0}\}\right)$ также является семипространством классической вьпуклости в $\mathbf{0}$. Исходя из свойств классических семипространств, множество $\mathbb{R}^{n} \backslash S_{0}$ является острьп выпукльм конусом, т.е. не содержашим прямых, которые проходят через его вершину. Справедливо $-O\left(S_{0}\right) \subseteq \mathbb{R}^{n} \backslash S_{0}$. Тогда $C H\left[-O\left(S_{0}\right)\right] \subseteq \mathbb{R}^{n} \backslash S_{0}$ и $C H\left[-O\left(S_{0}\right)\right]-$ острый 
выпукльй конус. Заметим, что $-O\left(S_{0}\right) \cup O\left(S_{0}\right)=O$. Для любого $v \in O$ выполняется утверждение: если $v \notin-O\left(S_{0}\right)$, то $-v \in-O\left(S_{0}\right)$. Тогда $C H\left[-O\left(S_{0}\right)\right]-$ максимальный острый конус, поскольку $C H\left[-O\left(S_{0}\right) \cup\{v\}\right]$ для любого $v \notin-O\left(S_{0}\right)$ из $O$ не является острым конусом, так как содержит как вектор $v$, так и $-v$. Тогда согласно теореме 3 из [1] и теореме 5 множество $\mathbb{R}^{n} \backslash C H\left[-O\left(S_{0}\right)\right]$ является $O C$-семипространством $S_{0}^{o c}$ в точке 0. Из $C H\left[-O\left(S_{0}\right)\right] \subseteq \mathbb{R}^{n} \backslash S_{0}$ следует, что $S_{0} \subseteq \mathbb{R}^{n} \backslash C H\left[-O\left(S_{0}\right)\right]$. Поскольку $C H\left[-O\left(S_{0}\right)\right] \subseteq \mathbb{R}^{n} \backslash S_{0}$, то $C H\left[-O\left(S_{0}\right)\right]+a \subseteq \mathbb{R}^{n} \backslash S_{0}$ для любого $a \in \mathbb{R}^{n} \backslash S_{0}$, так как $C H\left[-O\left(S_{0}\right)\right]$ и $\mathbb{R}^{n} \backslash S_{0}$ - выпуклые конусы. Следовательно, $\forall a \in \mathbb{R}^{n} \backslash S_{0}$ : $S_{0} \subseteq \mathbb{R}^{n} \backslash C H\left[-O\left(S_{0}\right)+a\right]$ и, значит,

$$
S_{0}=\bigcap_{a \in \mathbb{R}^{n} \backslash S_{0}} \mathbb{R}^{n} \backslash C H\left[-O\left(S_{0}\right)+a\right]
$$

Итак, мы представили семипространство $S_{0}$ в виде пересечения подходящих $O C$-семипространств. Аналогично, любое семипространство $S_{x}$ в произвольной точке $x \in \mathbb{R}^{n}$ может быть представлено как

$$
S_{x}=S_{0}+x=\bigcap_{a \in \mathbb{R}^{n} \backslash\left(S_{0}+x\right)} \mathbb{R}^{n} \backslash C H\left[-O\left(S_{0}\right)+a+x\right]=\bigcap_{a \in \mathbb{R}^{n} \backslash S_{x}} S_{a+x}^{o c} .
$$

То есть любое семипространство классической выпуклости в произвольной точке мы можем представить как пересечение $O C$-семипространств. Теорема доказана.

$O C$-выпуклую оболочку произвольного множества $A \subseteq \mathbb{R}^{n}$ (т.е. пересечение всех $O C$-вьпуклых множеств, содержащих $A$ ) обозначим через $\operatorname{conv}^{o c}[A]$. Далее мы будем рассматривать только такие множества $A$, для которых $\operatorname{conv}^{\text {oc }}[A] \neq \mathbb{R}^{n}$. Из теоремы 6 следует утверждение: $\operatorname{conv}^{o}[A] \subseteq \operatorname{conv}^{o c}[A] \subseteq \operatorname{conv}[A]$. Здесь $\operatorname{conv}[A]$ обозначает классически выпуклую оболочку множества $A$. Таким образом, $O C$-выпуклая оболочка является аппроксимацией $O$-выпуклой оболочки. Важную роль в определении $O C$-вьпуклых оболочек занимает понятие $O C$-экстремальной точки. Точку $a \in A$ назовем $O C$ - экстремальной для $A$, если $a \notin \operatorname{conv}^{o c}[A \backslash\{a\}]$. Совокупность всех $O C$-экстремальных точек для множества $A$ обозначим как $\operatorname{ext}^{o c}[A]$. Следовательно, $O C$-вьпуклую оболочку conv ${ }^{o c}[A]$ можно определить через $O C$-экстремальные точки следующим образом. Для любой точки $x \in \mathbb{R}^{n}$ вьполняется утверждение: $x \in \operatorname{conv}^{\text {oc }}[A]$ тогда и только тогда, когда $x \notin \operatorname{ext}^{o c}[A \cup\{x\}]$. Справедлива следующая теорема.

Теорема 7. Для множества $A \subseteq \mathbb{R}^{n}$ точка $x \in A$ будет ОС-әкстремальной для $A$ только в том случае, когда найдется в точке $x$ такое ОС-семипространство $S_{x}^{o c}$, что $A \backslash\{x\} \subseteq S_{x}^{o c}$.

ДокАЗАТЕЛЬСТво. Согласно теореме 5 для любого $O C$-вьпуклого множества $F$, не содержащего точку $x$, найдется $O C$-семипространство $S_{x}^{o c}$, включающее $F$. Из этого следует, что $x \in \operatorname{ext}^{o c}[A]$ тогда и только тогда, когда существует $O C$-семипространство $S_{x}^{o c}$ в точке $x$ такое, что conv ${ }^{o c}[A \backslash\{x\}] \subseteq S_{x}^{o c}$. Из этого следует $A \backslash\{x\} \subseteq S_{x}^{o c}$. Обратно, если $A \backslash\{x\} \subseteq S_{x}^{o c}$, то conv ${ }^{o c}[A \backslash\{x\}] \subseteq S_{x}^{o c}$ и, следовательно, $x \in \operatorname{ext}^{o c}[A]$. Теорема доказана. 
СлЕДСТВИЕ 1. Определение ОС-выпуклой оболочки через ОС-семипространства: точка $а \in \mathbb{R}^{n}$ принадлежит $\operatorname{conv}^{o c}[A]$, әде $A \subseteq \mathbb{R}^{n}$, только в том случае, когда для любого ОС-семипространства $S_{a}^{o c}$ в точке а существует точка $x \in A$ такая, что $x \notin S_{a}^{o c}$.

ТЕОрема 8. Если О конечно, то ОС-выпуклость конечно определена.

ДоказАтЕЛьство. Пусть задано бесконечное множество $A \subset \mathbb{R}^{n}$. Докажем, что для любого $a \in \operatorname{conv}^{o c}[A]$ существует конечное множество $B \subset A$ такое, что $a \in \operatorname{conv}^{o c}[B]$. Если $a \in A$, то $B=\{a\}$. Рассмотрим случай, когда $a \notin A$. Заметим, что $a \in \operatorname{conv}^{o c}[A]$, только если $a \notin \operatorname{ext}^{o c}[A \cup\{a\}]$. В таком случае для любого $O C$-семипространства $S_{a}^{o c}$ в точке $a$ множество $A$ не содержится в $S_{a}^{o c}$ в силу теоремы 7 , что эквивалентно утверждению $\forall S_{a}^{o c}(\exists x \in A)\left[x \notin S_{a}^{o c}\right]$. Поскольку количество $O C$-семипространств в точке $a$ конечно (из-за конечности множества $O$ ), то можно найти конечноеподмножество $B \subset A$ такое, что $\forall S_{a}^{o c}(\exists x \in B)\left[x \notin S_{a}^{o c}\right]$. Это означает, что $a \notin \operatorname{ext}^{o c}[B \cup\{a\}]$ и, следовательно, $a \in \operatorname{conv}^{o c}[B]$. Теорема доказана.

Остается открытым вопрос о конечной определенности $O C$-выпуклости в случае, когда $O$ бесконечно.

Для $O C$-вьпуклых множеств справедлива следующая теорема, аналогичная теореме Крейна-Мильмана для классически выпуклых множеств.

Теорема 9. Пусть О конечно. Если $A \subset \mathbb{R}^{n}-$ компакт, то

$$
\operatorname{conv}^{o c}[A]=\operatorname{conv}^{o c}\left[\operatorname{ext}^{o c}[A]\right] \text {. }
$$

ДокАЗАтЕЛЬСтво. Обозначим через $(x, y)$ скалярное произведение векторов $x, y \in \mathbb{R}^{n}$. Доказательство будем вести от противного. Предположим, что существует компактное множество $A \subset \mathbb{R}^{n}$ такое, что $\operatorname{conv}^{o c}\left[\operatorname{ext}^{o c}[A]\right] \neq \operatorname{conv}^{o c}[A]$. Тогда найдется точка $x \in A$ такая, что $x \notin \operatorname{conv}^{o c}\left[\operatorname{ext}^{o c}[A]\right]$. Следовательно, $x \in \operatorname{ext}^{o c}\left[\operatorname{ext}^{o c}[A] \cup\{x\}\right]$. Значит, отьпется $O C$-семипространство $S_{x}^{o c}=\mathbb{R}^{n} \backslash(C+x)$ такое, что $\operatorname{ext}^{o c}[A] \subseteq S_{x}^{o c}$ или, что то же самое, $(C+x) \cap \operatorname{ext}^{o c}[A]=\varnothing$. Так как $O$ конечно, то $C+x$ - замкнутое многогранное множество с угловой точкой $x$. Следовательно, существует замкнутое полупространство $H=\left\{v \in \mathbb{R}^{n} \mid(a, v) \geqslant \alpha\right\}$, где $a \in \mathbb{R}^{n}$ и $\alpha \in \mathbb{R}$ - постоянные, такое, что $\left\{v \in \mathbb{R}^{n} \mid(a, v)=\alpha\right\} \cap(C+x)=\{x\}$ и $C+x \subset H$. Иньми словами, найдется гиперплоскость $\left\{v \in \mathbb{R}^{n} \mid(a, v)=\alpha\right\}$, которая пересекается с многогранньм множеством $C+x$ только в угловой точке $x$ (см. [3]). Рассмотрим следующую задачу:

$$
(a, v) \rightarrow \max , \quad v \in(C+x) \cap A .
$$

Заметим, что так как $C+x$ замкнуто, то $(C+x) \cap A-$ компакт. Значит, по теореме Вейерштрасса функция $(a, v)$ принимает максимальное значение на $(C+x) \cap A$ и, следовательно, задача (1) имеет решение. Зафиксируем какую-нибудь точку $v^{*}$, на которой достигается максимум $(a, v)$. Рассмотрим множество $C+v^{*}$. Полупространство $H^{*}=\left\{v \in \mathbb{R}^{n} \mid(a, v) \geqslant\left(a, v^{*}\right)\right\}$ будет целиком содержать множество $C+v^{*}$, так как $H$ целиком содержит $C+x$. С другой стороны, $\left\{v \in \mathbb{R}^{n} \mid(a, v)=\left(a, v^{*}\right)\right\} \cap\left(C+v^{*}\right)=\left\{v^{*}\right\}$. Поскольку для любой точки $v \in(C+x) \cap A$ справедливо $(a, v) \leqslant\left(a, v^{*}\right)$, то $((C+x) \cap A) \cap$ $\left(C+v^{*}\right)=\left\{v^{*}\right\}$. Из $C+v^{*} \subseteq C+x$ следует $A \cap\left(C+v^{*}\right)=\left\{v^{*}\right\}$, т.е. $v^{*} \in$ ext $^{o c}[A]$. Но это противоречит принятому ранее условию $(C+x) \cap \operatorname{ext}^{o c}[A]=\varnothing$. Теорема доказана. 
СлЕДСТВИЕ 2. Пусть О бесконечно. Если $A \subset \mathbb{R}^{n}$ - конечное множество точек, mo conv ${ }^{o c}[A]=\operatorname{conv}^{o c}\left[\operatorname{ext}^{o c}[A]\right]$.

ДокАЗАтЕльство. Как следует из доказательства теоремы 7 , если $\operatorname{conv}^{o c}\left[\operatorname{ext}^{o c}[A]\right]$ $\subset \operatorname{conv}^{o c}[A]$, то найдется $x \in A$ такая, что $(C+x) \cap \operatorname{ext}^{o c}[A]=\varnothing$. Тогда $x \notin \operatorname{ext}^{o c}[A]$. Так как $A$ конечно, то $(C+x) \cap A$ конечно. Из этого следует, что мы можем найти такую точку $y \in(C+x) \cap A$, что $C+y \subset C+x$ и $(C+y) \cap((C+x) \cap A)=\{y\}$. Следовательно, $(C+y) \cap A=\{y\}$. Тогда $y \in \operatorname{ext}^{o c}[A]$. Получаем противоречие с $(C+x) \cap \operatorname{ext}^{o c}[A]=\varnothing$. Следствие доказано.

Перейдем теперь к вопросу вычислительной сложности нахождения $O C$-вьпукльх оболочек.

ТЕОРема 10. Пусть произвольно заданы размерность п линейного пространства, конечное множсество ненулевых векторов (направлений) $O \subset \mathbb{R}^{n}$, конечное множество точек $A \subset \mathbb{R}^{n}$ и выбрана некоторая точка $а \in A$. Тогда проблема распознавания принадлежсности точки а кмножеству $\operatorname{ext}^{\text {ос }}[A] \mathrm{NP}$-полна.

ДокАЗАТЕЛЬСТво. Покажем, как полиномиально свести проблему выполнимости КН $\Phi$ к данной задаче. Пусть задана произвольная $\mathrm{KH} \Phi F=\bigwedge_{i=1}^{p} C_{i}$, где $C_{i}-$ некоторая дизъюнкция литералов из множества $\left\{x_{1}, \bar{x}_{1}, \ldots, x_{n}, \bar{x}_{n}\right\}$. Допустим, что $O=$ $\left\{e_{1},-e_{1}, \ldots, e_{n},-e_{n}\right\}$, где векторы $e_{1}, e_{2}, \ldots, e_{n}$ образуют базис в пространстве $\mathbb{R}^{n}$. Пусть $A=\left\{a_{i} \mid i \in \overline{0, p}\right\}$, где $a_{0}=\mathbf{0}$ - начало координат, $a_{i}=\alpha_{1}^{(i)} e_{1}+\cdots+\alpha_{n}^{(i)} e_{n}$ $\forall i \in \overline{1, p}$. Коэффициенты $\alpha_{1}^{(i)}, \ldots, \alpha_{n}^{(i)}$ определяются следующим образом: $\alpha_{j}^{(i)}=-1$, если литерал $x_{j}$ входит в $C_{i} ; \alpha_{j}^{(i)}=1$, если литерал $\bar{x}_{j}$ входит в $C_{i} ; \alpha_{j}^{(i)}=0$, если ни $x_{j}$, ни $\bar{x}_{j}$ не входят в $C_{i}$. Докажем, что точка $a_{0}$ принадлежит $\operatorname{ext}^{o c}[A]$ тогда и только тогда, когда КН $\Phi$ вьполнима. Тогда для любого половинного множества $M \subset O$ множество $\mathbb{R}^{n} \backslash C H[M]$ будет семипространством $O C$-вьпуклости в точке $a_{0}$. Кроме того, каждой интерпретации булевых переменных $x_{1}, \ldots, x_{n}$ можно взаимно-однозначно поставить в соответствие некоторое половинное множество $M\left(x_{1}, \ldots, x_{n}\right)=\left\{e_{1}\left(x_{1}\right), \ldots, e_{n}\left(x_{n}\right)\right\}$, где $e_{j}\left(x_{j}\right)=e_{j}, j \in \overline{1, n}$, если $x_{j}=1$, и $e_{j}\left(x_{j}\right)=-e_{j}$, если $x_{j}=0$. Заметим, что если имеются значения переменных $x_{1}, \ldots, x_{n}$ такие, что $a_{i} \notin C H\left[M\left(x_{1}, \ldots, x_{n}\right)\right]$, где $i \geqslant 1$ (или $\left.a_{i} \in \mathbb{R}^{n} \backslash C H\left[M\left(x_{1}, \ldots, x_{n}\right)\right]\right)$, то найдется $\alpha_{j}^{(i)} \neq 0$ такое, что $\alpha_{j}^{(i)}=-1$, если $e_{j}\left(x_{j}\right)=e_{j}$, и $\alpha_{j}^{(i)}=1$, если $e_{j}\left(x_{j}\right)=-e_{j}$. Но тогда дизъюнкт $C_{i}$ будет вьполняться при этих значениях $x_{1}, \ldots, x_{n}$. Итак, если существуют значения переменных $x_{1}, \ldots, x_{n}$, при которых $A \backslash\left\{a_{0}\right\} \subseteq \mathbb{R}^{n} \backslash C H\left[M\left(x_{1}, \ldots, x_{n}\right)\right]$, то это означает, что КН $\Phi F=\bigwedge_{i=1}^{p} C_{i}$ выполняется при этих $x_{1}, \ldots, x_{n}$. Утверждение $A \backslash\left\{a_{0}\right\} \subseteq \mathbb{R}^{n} \backslash C H\left[M\left(x_{1}, \ldots, x_{n}\right)\right]$ эквивалентно $a_{0} \in \operatorname{ext}^{o c}[A]$. Таким образом, осуществлено полиномиальное сведение проблемы выполнимости КН $\Phi$ к указанной в теореме задаче. Теперь покажем, что произвольная проблема определения $x \in \operatorname{ext}^{o c}[A]$ принадлежит к классу NP. Заметим, что $x \in \operatorname{ext}^{o c}[A]$ эквивалентно утверждению $(\exists M \subset O)(\forall y \in A \backslash\{x\})\left[y \in \mathbb{R}^{n} \backslash C H[M], M \cup\right.$ $-M=O$ и $M \cap-M=\varnothing]$ в силу теоремы 7. Выражение $(\forall y \in A \backslash\{x\})\left[y \in \mathbb{R}^{n} \backslash C H[M]\right.$, $M \cup-M=O$ и $M \cap-M=\varnothing]$ проверяется за полиномиальное время по длине входа $|O|+|A|$ для любого $M \subset O$. Из этого следует принадлежность задачи $x \in \operatorname{ext}^{o c}[A] \mathrm{k}$ классу NP. Теорема доказана.

СлЕДСТВИЕ 3. Проблема распознавания принадлежности произвольной точки $x$ из $A \kappa \operatorname{conv}^{o c}[A \backslash\{x\}]$ coNP-полна. 
Таким образом, проблема вычисления $O C$-выпуклых оболочек достаточна сложна. Кроме того, мы можем частично ответить на вопрос И. Матоушека и П. Плехача [2], а именно: если задано некоторое "разумное" семейство множеств точек $\mathscr{Q}$ из $\mathbb{R}^{n}$, то как эффективно вычислить $\mathscr{Q}$-оболочку конечного множества $X$ (т.е. пересечение всех множеств из $\mathscr{Q}$, содержащих $X)$ ? Как следует из теоремы 10 , если $\mathscr{Q}=O C$, то задача вычисления $\mathscr{Q}$-оболочек является NP-трудной даже в случае выпуклости, порожденной пересечением дополнений ортантов. Однако, если мы зафиксируем размерность линейного пространства, например $\mathbb{R}^{3}$, то определенные в теореме 10 и ее следствии 3 проблемы становятся полиномиально разрешимыми. Действительно, чтобы определить будет ли точка $a \in A$ принадлежать $\operatorname{conv}^{o c}[A]$, в силу следствия 1 теоремы 7 достаточно знать все $O C$-семипространства $S_{a}^{o c}$ (или в силу теоремы 5 все $C$-семипространства $O$-вьпуклости в точке $a)$. Все $O C$-семипространства в точке $a$ можно найти алгоритмом ENUMCONE. Число $O C$-семипространств по теореме 4 можно оценить как $O\left(m^{(n+1)(n-2) / 2}\right)$, т.е. как полином по $m$, если размерность $n$ фиксирована. В то же время алгоритм ENUMCONE по теореме 3 обладает полиномиальной скоростью. Итак, общее время перечисления всех $O C$-семипространств в точке $a$ полиномиально по входу алгоритма ENUMCONE. Очевидно, что все остальные необходимые проверки также занимают полиномиальное время.

\section{СПИСОК ЦИТИРОВАННОЙ ЛИТЕРАТУРЫ}

[1] Метельский Н. Н., Мартынчик В.Н. Частичная вьпуклость // Матем. заметки. 1996. T. 60. № 3. C. 406-413.

[2] Matousek J., Plechac P. On functional separately convex hulls // KAM Series. Technical Report № 95-292. Prague: Charles Univ., 1995.

[3] Лейхтвейс К. Выпуклые множества. М.: Наука, 1985.

[4] Seymour P. D. A note on hyperplane generation // J. Combinatorial Theory. Ser. B. 1994. P. 88-91.

[5] Солтан В. П. Введение в аксиоматическую теорию выпуклости. Кишинев: Штиинца, 1984.

Институт математики НАН Беларуси, г. Минск

Поступило

E-mail: naidenko@im.bas-net.by

02.11 .1998 